\title{
AN ALGORITHM TO DEVELOP LUMPED MODEL FOR GUNN-DIODE DYNAMICS
}

\author{
UMESH KUMAR \\ Department of Electrical Engineering, I.I.T., Hauz Khas, \\ New Delhi -110 016, India
}

(Received 12 November 1997; In final form 23 January 1998)

A nonlinear lumped model can be developed for Gunn-Diodes to describe the diffusion effects as the domain travels from cathode to anode of a Gunn-Diode. The model describes the domain extinction and nucleation phenomena. It allows the user to specify arbitrary nonlinear drift velocity $V(E)$ and nonlinear diffusion $D(E)$.

The model simulates arbitrary Gunn-Diode circuits operating in any matured high field domain or in the LSA mode.

Here we have constructed an algorithm to lead to development of this model.

Keywords: Algorithm; lumped model; nonlinear dynamics; Gunn-Diode

\section{INTRODUCTION}

Nonlinearity is an inherent property of semi conductor devices. A device is called nonlinear when the relationship between its terminal variables namely voltage and current is other than linear. Modern device like the Gunn-Diode is strongly nonlinear. These devices exhibit complex dynamic behaviour.

With the leaping advances in integrated circuits the boundaries between circuit design and device design are becoming less recognizable. Therefore, in addition to the understanding of terminal behaviour of a device, it also becomes important to understand the interrelationship among physical processes, device geometry and terminal behaviour. 


\section{LUMPED NONLINEAR CIRCUIT MODEL}

Let us take a 1-D structure of a Gunn-Diode of length $L$, area $A$, dielectric constant $\varepsilon$ and a uniform donor concentration $n_{o} . n_{o} L>10^{12}$ enables to support high field domains consisting of an accumulation layer with $n>n_{o}$ and a depletion layer with carrier concentration $n<n_{o}$. As the domain grows in size, it propagates from the cathode $(X=O)$ to the anode $(X=L)$ with an instantaneous velocity $V_{D}(t)$.

Shapes of $n(x)$ and the field distribution (due to the dipole induction) $E(X)$ at different instants of time are governed by complex dynamics and affect device current and voltage.

\section{DESCRIPTION OF THE MODEL}

1. Cathode to anode capacitance:

$$
C_{1}=\frac{\varepsilon A}{L}
$$

2. Domain capacitance:

$$
C_{2}=\frac{\varepsilon A}{W}
$$

where $W=$ Average domain width

$$
=\underset{T \rightarrow \infty}{L t} \int_{0}^{T}\left|X_{2}(t)-X_{1}(t)\right| d t
$$

3. Nonlinear Resistor: $R$ with current $I_{R}$ and Voltage $V_{R}$

$$
I_{R}=G\left(V_{R}\right)=A q n_{o} V\left(V_{R} / L\right)
$$

where $V(E)=V\left(V_{R} / L\right)$ and $E=V_{R} / L$

4. Nonlinear current controlled source:

$$
\begin{aligned}
I_{D} & =I_{D}\left(V_{1}, V_{2}, I\right) \\
& =C_{2} F\left(V_{1}, V_{2}\right)-I
\end{aligned}
$$


where

$$
\begin{aligned}
F\left(V_{1}, V_{2}\right)= & n o\left\{\int_{V_{1} / L}^{E_{m}}\left|\frac{V\left(V_{1} / L\right)-V(E)}{n_{a}(E)-n_{o}}\right| d E\right. \\
& \left.+\int_{V_{1} / L}^{E_{m}}\left|\frac{V\left(V_{1} / L\right)-V(E)}{n_{o}-n_{d}(E)}\right| d E\right\}
\end{aligned}
$$

$n_{a}(E), n_{d}(E), E_{m}$ are found for each value of $\left(V_{1}, V_{2}\right)$ as:

$$
\begin{gathered}
g\left(E_{m} ; V_{1}, V_{2}\right)=0 \\
g\left(E_{m} ; V_{1}, V_{2}\right)=V_{2}-\frac{\varepsilon}{q}\left\{\int_{V_{1} / L}^{E_{m}} \frac{E-V_{1} / L}{n_{a}(E)-n_{o}} d E+\int_{V_{1} / L}^{E_{m}} \frac{E-V_{1} / L}{n_{o}-n_{d}(E)} d E\right\}
\end{gathered}
$$

5. Accumulation layer carrier conc. $n_{a}(E)$ :

For each given value of $\left(V_{1}, V_{2}\right), n_{a}(E)$ is obtained by solving the following scalar nonlinear ordinary differential equation:

$$
\frac{d n_{a}(E)}{d E}=\mathrm{Na}\left(n_{a}, E ; V_{1}, V_{d}\right)
$$

where with $D(E)$ being a nonlinear function of $E, \mathrm{Na}\left(n_{a}, E ; V_{1}, V_{D}\right)=$

$$
\begin{gathered}
\frac{n_{a}\left[V(E)-V_{D}\right]+n_{o}\left[V_{D}-V\left(V_{1} / L\right)\right]-q / \varepsilon\left(n_{a}-n_{o}\right) n_{a} D^{\prime}(E)}{q / \varepsilon\left(n_{a}-n_{o}\right) D(E)} \\
\left.n_{a}(E)\right|_{E=E_{o}}=V_{1} / L=n_{o} \\
\left.n_{a}(E)\right|_{E=E_{m}}=n_{o}
\end{gathered}
$$

$V(D)=V_{D}\left(V_{1}, V_{2}\right)$ is domain velocity.

6. Depletion layer concentration: $n_{d}(E)$ : For each given value of $\left(V_{1}\right.$, $\left.V_{2}\right), n_{d}(E)$ is obtained by solving the initial value problem:

$$
d \frac{n_{d}}{d E}=N_{d}\left(n_{d}, E ; V_{1}, V_{D}\right)
$$




$$
\begin{aligned}
& \mathrm{N}_{d}\left(n_{d}, E ; V_{1}, V_{D}\right) \\
& =\frac{n_{d}\left[V(E)-V_{D}\right]+n_{o}\left[V_{D}-V\left(V_{1} / L\right)\right]-(q / \varepsilon)\left(n_{d}-n_{o}\right) n_{d} D^{\prime}(E)}{(q / \varepsilon)\left(n_{d}-n_{o}\right) D(E)}
\end{aligned}
$$

where

$$
\begin{gathered}
\left.n_{d}(E)\right|_{E=E_{o}}=V_{1} / L=n_{o} \\
V(D)=V_{D}\left(V_{1}, V_{2}\right)
\end{gathered}
$$

$V_{D}$ is already obtained from Eq. (8a) for given $\left(V_{1}, V_{2}\right)$

$n_{d}(E)$ depends on both $V_{1}$ and $E$ and so $n_{d}(E)=n_{d}\left(E ; V_{1}, E_{m}\right)$.

Eqs. (7), (8) and (9) are coupled together. Hence we must solve them as simultaneous equations by iterative methods.

Eq. (7) is only a scalar algebraic equations and can be solved by Newton-Raphson or the Secant method.

Once $n_{a}(E), n_{d}(E)$ and $E_{m}$ are found, the function $F\left(V_{1}, V_{2}\right)$ can be found by the help of the following algorithm:

$$
\frac{\text { Algorithm for Computing } F\left(V_{1}, V_{2}\right)}{\operatorname{Given}\left(V_{1}, V_{2}\right)=\left(\bar{V}_{1}, \bar{V}_{2}\right)}
$$

Step 1 Substitute $\left(\bar{V}_{1}, \bar{V}_{2}\right)$ into Eqs. (7), (8) and (9).

Step 2 Assume two initial quesses $E_{m}^{(0)}$ and $E_{m}^{(1)}$

Step 3 Let $E_{m}=E_{m}^{(k)}$ and solve the two point boundary value problem (8) for

$$
\begin{gathered}
V_{D}=V_{D}^{(k)}\left(\bar{V}_{1}, \bar{V}_{2}\right)=V_{D}^{(k)}, \quad k=0,1 \\
n_{a}=n_{a}\left(E ; \bar{V}_{1}, E_{m}^{(k)}\right) \\
=n_{a}{ }^{(k)}(E), \quad k=0,1
\end{gathered}
$$

Step 4 Substitute (i) for $V D$ in 9 (b) with $k=0$ and $k=1$, respsectively and solve the initial value problem for

$$
n_{d}=n_{d}\left(E ; \bar{V}_{1}, E_{m}^{(k)}\right)=n_{d}^{(k)}(E), \quad k=0,1
$$


Step 5 Substitute (ii) and (iii) in (7) and solve for $E_{m}^{k+1}$ using the following secant iteration formula:

$$
E_{m}^{(j+1)}=E_{m}^{(j)}-F\left(E_{m}^{(j)}\right) g\left(E_{m}^{(j)}, \bar{V}_{1}, \bar{V}_{2}\right)
$$

where

$$
F\left(E_{m}^{(j)}\right)=\frac{E_{m}^{(j)}-E_{m}^{(j-1)}}{g\left(E_{m}^{(j)}, \bar{V}_{1}, \bar{V}_{2}\right)-g\left(E_{m}^{(j-1)}, \bar{V}_{1}, \bar{V}_{2}\right)}
$$

Step 6 Iterate steps (3) through (5) with the subscript ' $k$ ' replaced by $j$, $j=2,3 \ldots$ until the interation converges, namely

$$
\begin{gathered}
n_{a}^{(j)}(E) \longrightarrow n_{a}\left(E ; \bar{V}_{1}, \bar{V}_{2}\right) \\
n_{d}^{(j)}(E) \longrightarrow n_{d}\left(E ; \bar{V}_{1}, \bar{V}_{2}\right)
\end{gathered}
$$

and

$$
E_{m}^{(j)} \longrightarrow E_{m}^{(j)}\left(\bar{V}_{1}, \bar{V}_{2}\right)
$$

Step 7 Substitute (vi), (vii) and (viii) for $n_{a}(E), n_{d}(E)$ and $E_{m}$ in Eq. (5) and compute the two integrals numerically to obtain the required $F\left(\bar{V}_{1}, \bar{V}_{2}\right)$.

\section{References}

[1] Bulkman, P. J., Hobson, G. S. and Taylor, B. G. (1972). Transferred Electron Devices, Academic Press, New York.

[2] Hartanagal, H. L. (1973). Gunn Effect Logic Devices, Heinemann Educational Books, London.

[3] Hobson, G. S. (1974). The Gunn Effect, Clarendon Press, Oxford.

[4] Gunshor, R. L. and Kak, A. C. (1972). "Lumped Circuit representation of GunnDiodes in domain mode", IEEE Trans. Electron Devices, ED-19, 765-770.

[5] Kak, A. C. and Gunshor, R. L. (1973). "The Transient behaviour of high field dipole domains in transferred electron devices", IEEE Trans. Electron Devices, ED20, $1-5$.

[6] Butcher, P. N. (1967). "The Gunn Effect", Report on progress in physics, XXX, (part. I), 47-148. 

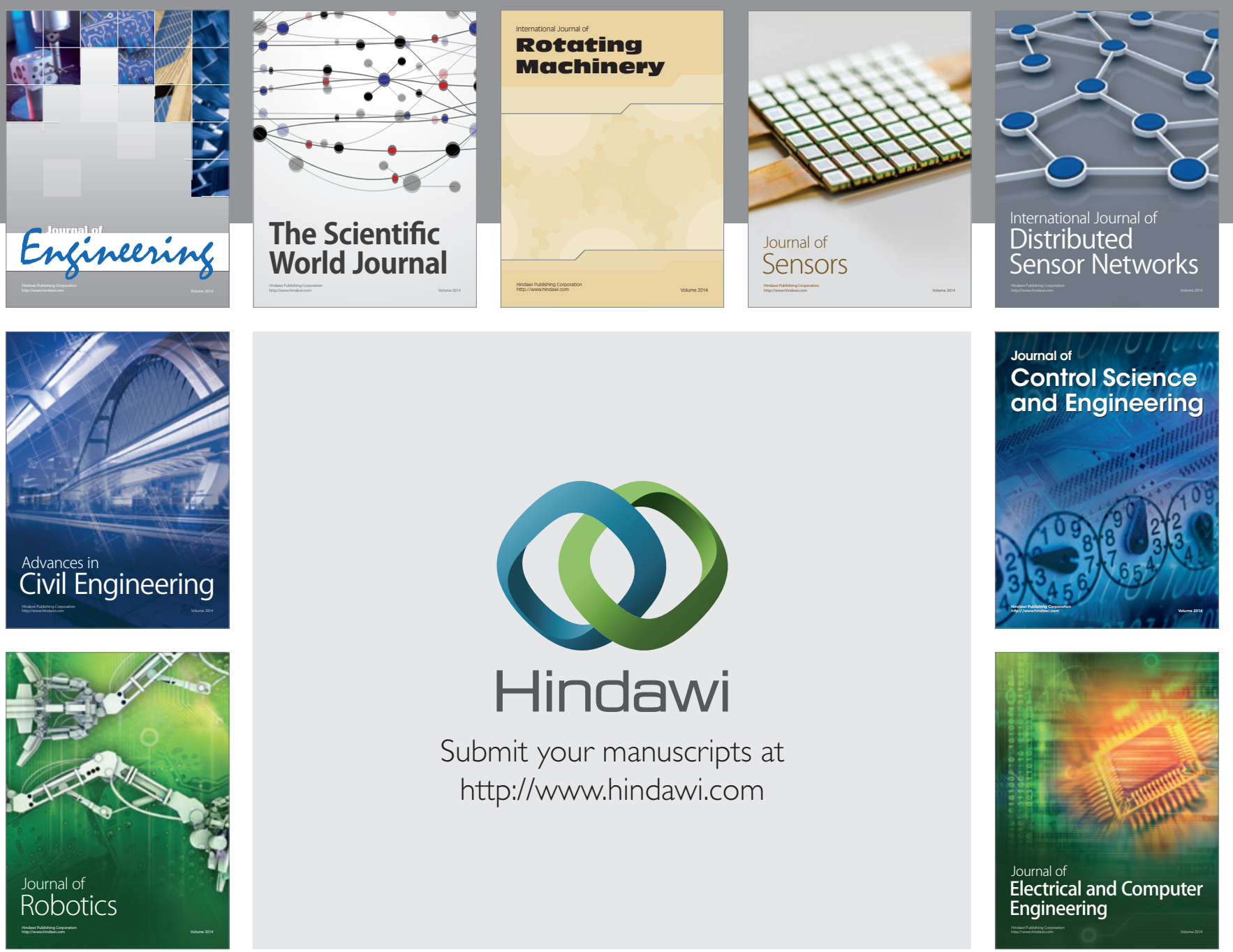

Submit your manuscripts at

http://www.hindawi.com
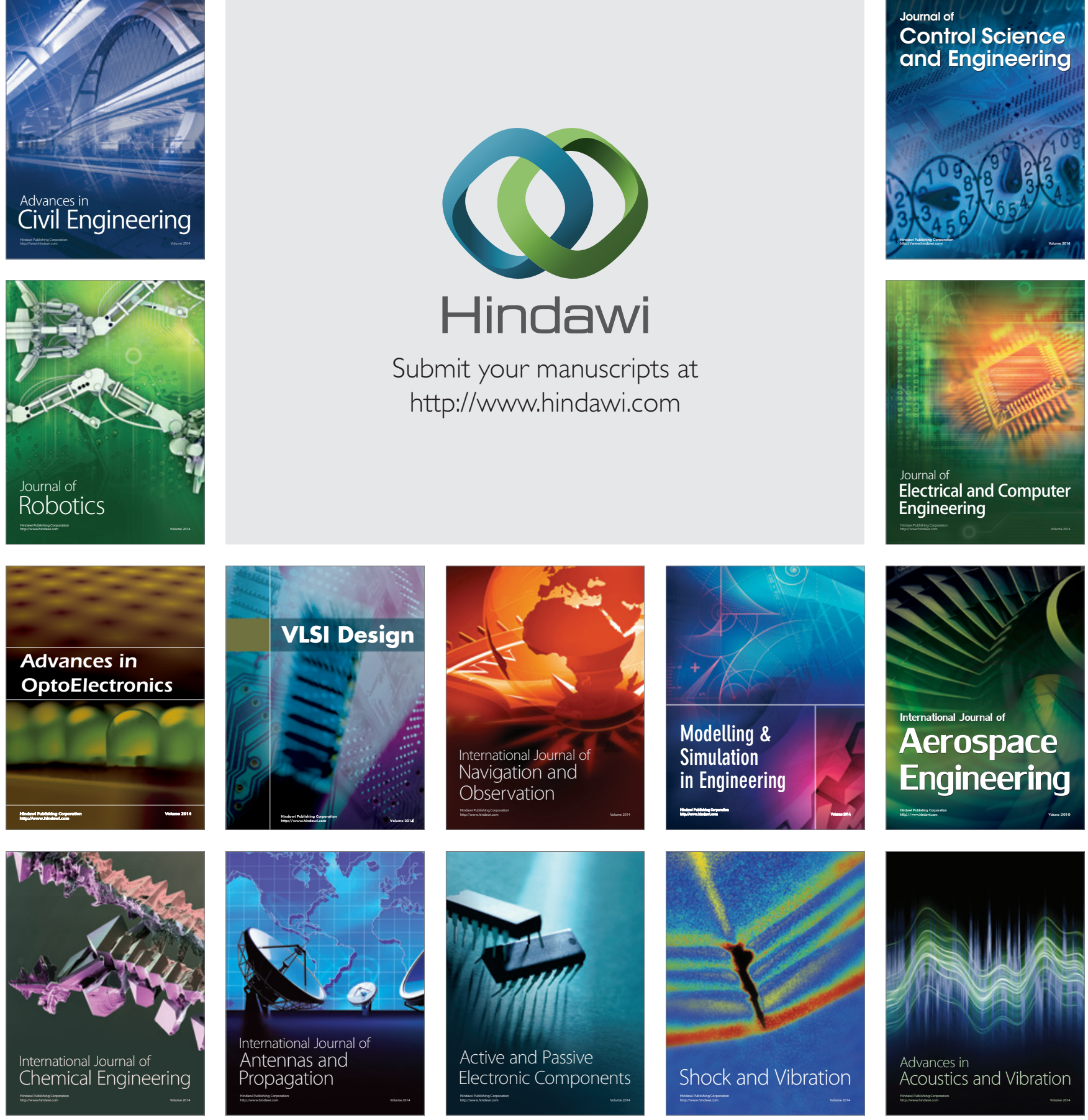